\title{
Hamiltonian formalism for solving the Vlasov-Poisson equations and its applications to periodic focusing systems and coherent beam-beam interaction
}

\author{
Stephan I. Tzenov and Ronald C. Davidson \\ Plasma Physics Laboratory, Princeton University, Princeton, New Jersey 08543
}

(Received 3 October 2001; published 4 February 2002)

\begin{abstract}
A Hamiltonian approach to the solution of the Vlasov-Poisson equations has been developed. Based on a nonlinear canonical transformation, the rapidly oscillating terms in the original Hamiltonian are transformed away, yielding a new Hamiltonian that contains slowly varying terms only. The formalism has been applied to the dynamics of an intense beam propagating through a periodic focusing lattice, and to the coherent beam-beam interaction. A stationary solution to the transformed Vlasov equation has been obtained.
\end{abstract}

DOI: 10.1103/PhysRevSTAB.5.021001

PACS numbers: $29.20 .-\mathrm{c}, 41.20 . \mathrm{Cv}, 41.20 . \mathrm{Jb}$

\section{INTRODUCTION}

The evolution of charged particle beams in accelerators and storage rings can often be described by the VlasovMaxwell equations [1,2]. At high energies the discreteparticle collision term [3] comprises a small correction to the dynamics and can be neglected. Radiation effects at sufficiently high energies for leptons can be a significant feature of the dynamics, and should normally be included in the model under consideration.

The Vlasov-Maxwell equations constitute a considerable simplification in the description of charged particle beam propagation. Nonetheless, there are only a few cases that are tractable analytically $[1,2]$. Therefore, it is of utmost importance to develop a systematic perturbation approach that is able to provide satisfactory results in a wide variety of cases of physical interest.

Particle beams are subject to external forces that are often rapidly oscillating, such as quadrupolar focusing forces, rf fields, etc. In addition, the collective self-field excitations can be rapidly oscillating as well. A typical example is a colliding-beam storage ring device, where the evolution of each beam is strongly affected by the electromagnetic force produced by the counterpropagating beam [4-6]. The beam-beam kick each beam experiences is localized only in a small region around the interaction point and is periodic with a period of one turn.

In this and other important applications, one is primarily interested in the longtime behavior of the beam, thus discarding the fast processes on time scales of the order of the period of the rapid oscillations. To extract the relevant information, an efficient method of averaging is developed in Sec. II. Unlike the standard canonical perturbation technique [7-9], the approach used here is carried out in a "mixed" phase space (old coordinates and new canonical momenta [10]), which is simpler and more efficient in a computational sense. It should be pointed out that the formalism developed here is, strictly speaking, noncanonical and, in general, does not provide complete elimination of fast oscillating terms in the transformed Vlasov equation in the mixed variables. Nevertheless, such an elimination can be performed in the new Hamiltonian in the mixed variables. Moreover, if the distribution function is assumed to be an arbitrary function of the new time-independent Hamiltonian, it is a stationary solution of the nonlinear Vlasov equation in the mixed variables. The canonical perturbation method developed in this paper is further applied to intense beam propagation in a periodic focusing structure (Sec. III) and to the coherent beam-beam interaction (Secs. IV and V). A coupled set of nonlinear integral equations for the equilibrium beam densities is derived. As applied to the beam-beam interaction, the present formalism has been developed for collisionless systems and is intended to complement and extend previous models [11] that calculate quasiequilibrium states in the presence of damping and quantum fluctuations.

To summarize, the effectiveness of the Hamiltonian formalism developed in this paper is demonstrated in two particular examples. In the first example, discussed in Sec. III, the short-scale dynamics is contained in the external focusing force acting on the beam, while an essential feature of the coherent beam-beam interaction treated in Secs. IV and $\mathrm{V}$ is the relatively fast variation of the collective interaction between the colliding beams. The simplicity in applying the Hamiltonian averaging technique is embedded in the use of mixed canonical variables. Instead of expanding the generating function and the new Hamiltonian in terms of the new canonical coordinates and momenta $[7,8]$, one has to simply solve the Hamilton-Jacobi equations order by order. It should be emphasized that the mixed variable Hamiltonian formalism can be used to derive amplitude equations, describing processes of formation of patterns and coherent structures in a number of plasma and beam systems in which collective processes are important.

\section{THE HAMILTONIAN FORMALISM}

We consider an $N$-dimensional dynamical system, described by the canonical conjugate pair of vector variables $(\mathbf{q}, \mathbf{p})$ with components 


$$
\mathbf{q}=\left(q_{1}, q_{2}, \ldots, q_{N}\right), \quad \mathbf{p}=\left(p_{1}, p_{2}, \ldots, p_{N}\right) .
$$

The Vlasov equation for the distribution function $f(\mathbf{q}, \mathbf{p} ; t)$ can be expressed as

$$
\frac{\partial f}{\partial t}+[f, H]_{\mathbf{q}, \mathbf{p}}=0
$$

where

$$
[F, G]_{\mathbf{q}, \mathbf{p}}=\frac{\partial F}{\partial q_{i}} \frac{\partial G}{\partial p_{i}}-\frac{\partial F}{\partial p_{i}} \frac{\partial G}{\partial q_{i}}
$$

is the Poisson bracket, $H(\mathbf{q}, \mathbf{p} ; t)$ is the Hamiltonian of the system, and summation over repeated indices is implied. Next we define a canonical transformation via the generating function of the second type according to

$$
S=S(\mathbf{q}, \mathbf{P} ; t),
$$

and assume that the Hessian matrix

$$
\hat{\mathcal{H}}_{i j}(\mathbf{q}, \mathbf{P} ; t)=\frac{\partial^{2} S}{\partial q_{i} \partial P_{j}}
$$

of the generating function $S(\mathbf{q}, \mathbf{P} ; t)$ is nondegenerate, i.e.,

$$
\operatorname{det}\left(\hat{\mathcal{H}}_{i j}\right) \neq 0 .
$$

This implies that the inverse matrix $\hat{\mathcal{H}}_{i j}^{-1}$ exists. The new canonical variables $(\mathbf{Q}, \mathbf{P})$ are defined by the canonical transformation as

$$
p_{i}=\frac{\partial S}{\partial q_{i}}, \quad Q_{i}=\frac{\partial S}{\partial P_{i}} .
$$

We also introduce the distribution function defined in terms of the new canonical coordinates $(\mathbf{Q}, \mathbf{P})$ and the mixed pair of canonical variables $(\mathbf{q}, \mathbf{P})$ according to

$$
\begin{gathered}
f_{0}(\mathbf{Q}, \mathbf{P} ; t)=f(\mathbf{q}(\mathbf{Q}, \mathbf{P} ; t), \mathbf{p}(\mathbf{Q}, \mathbf{P} ; t) ; t), \\
F_{0}(\mathbf{q}, \mathbf{P} ; t)=f(\mathbf{q}, \mathbf{p}(\mathbf{q}, \mathbf{P} ; t) ; t) .
\end{gathered}
$$

In particular, in Eq. (8) the old canonical variables are expressed in terms of the new ones, which is ensured by the implicit function theorem, provided the relation (6) holds. As far as the function $F_{0}(\mathbf{q}, \mathbf{P} ; t)$ is concerned, we simply replace the old momentum $\mathbf{p}$ by its counterpart taken from the first of Eqs. (7). Because

$$
\frac{\partial p_{i}}{\partial P_{j}}=\frac{\partial^{2} S}{\partial q_{i} \partial P_{j}}=\hat{\mathcal{H}}_{i j} \Longrightarrow \frac{\partial P_{i}}{\partial p_{j}}=\hat{\mathcal{H}}_{i j}^{-1}
$$

we can express the Poisson bracket in terms of the mixed variables in the form

$$
[F, G]_{\mathbf{q}, \mathbf{P}}=\hat{\mathcal{H}}_{j i}^{-1}\left(\frac{\partial F}{\partial q_{i}} \frac{\partial G}{\partial P_{j}}-\frac{\partial F}{\partial P_{j}} \frac{\partial G}{\partial q_{i}}\right) .
$$

Differentiation of Eqs. (7) with respect to time $t$, keeping the old variables $(\mathbf{q}, \mathbf{p})$ fixed, yields

$$
\begin{gathered}
\frac{\partial^{2} S}{\partial q_{i} \partial t}+\frac{\partial^{2} S}{\partial q_{i} \partial P_{j}}\left(\frac{\partial P_{j}}{\partial t}\right)_{q p}=0, \\
\left(\frac{\partial Q_{i}}{\partial t}\right)_{q p}=\frac{\partial^{2} S}{\partial P_{i} \partial t}+\frac{\partial^{2} S}{\partial P_{i} \partial P_{j}}\left(\frac{\partial P_{j}}{\partial t}\right)_{q p},
\end{gathered}
$$

or

$$
\left(\frac{\partial P_{j}}{\partial t}\right)_{q p}=-\hat{\mathcal{H}}_{j i}^{-1} \frac{\partial^{2} S}{\partial q_{i} \partial t} .
$$

Our goal is to express the Vlasov equation (2) in terms of the mixed variables $(\mathbf{q}, \mathbf{P})$. Taking into account the identities

$$
\begin{gathered}
\frac{\partial Q_{i}}{\partial q_{j}}=\frac{\partial^{2} S}{\partial q_{j} \partial P_{i}}=\hat{\mathcal{H}}_{j i} \Longrightarrow \frac{\partial q_{i}}{\partial Q_{j}}=\hat{\mathcal{H}}_{j i}^{-1}, \\
\frac{\partial f_{0}}{\partial Q_{i}}=\hat{\mathcal{H}}_{i j}^{-1} \frac{\partial F_{0}}{\partial q_{j}}
\end{gathered}
$$

and

$$
\frac{\partial f_{0}}{\partial P_{i}}=\frac{\partial F_{0}}{\partial P_{i}}-\frac{\partial f_{0}}{\partial Q_{j}} \frac{\partial^{2} S}{\partial P_{i} \partial P_{j}},
$$

we obtain

$$
\begin{aligned}
\left(\frac{\partial f}{\partial t}\right)_{q p} & =\frac{\partial f_{0}}{\partial t}+\frac{\partial f_{0}}{\partial Q_{i}}\left(\frac{\partial Q_{i}}{\partial t}\right)_{q p}+\frac{\partial f_{0}}{\partial P_{i}}\left(\frac{\partial P_{i}}{\partial t}\right)_{q p} \\
& =\frac{\partial F_{0}}{\partial t}+\hat{\mathcal{H}}_{j i}^{-1}\left(\frac{\partial F_{0}}{\partial q_{i}} \frac{\partial^{2} S}{\partial t \partial P_{j}}-\frac{\partial F_{0}}{\partial P_{j}} \frac{\partial^{2} S}{\partial t \partial q_{i}}\right) \\
& =\frac{\partial F_{0}}{\partial t}+\left[F_{0}, \frac{\partial S}{\partial t}\right]_{\mathbf{q}, \mathbf{P}} .
\end{aligned}
$$

Furthermore, using the relation

$$
[f, H]_{\mathbf{q}, \mathbf{p}}=\left[F_{0}, \mathcal{H}\right]_{\mathbf{q}, \mathbf{P}},
$$

where

$$
\mathcal{H}(\mathbf{q}, \mathbf{P} ; t)=H\left(\mathbf{q}, \nabla_{q} S ; t\right),
$$

we express the Vlasov equation in terms of the mixed variables according to

$$
\frac{\partial F_{0}}{\partial t}+\left[F_{0}, \mathcal{K}\right]_{\mathbf{q}, \mathbf{P}}=0
$$

where

$$
\mathcal{K}(\mathbf{q}, \mathbf{P} ; t)=\frac{\partial S}{\partial t}+H\left(\mathbf{q}, \nabla_{q} S ; t\right)
$$

is the new Hamiltonian.

For the distribution function $f_{0}(\mathbf{Q}, \mathbf{P} ; t)$, depending on the new canonical variables, we clearly obtain

$$
\frac{\partial f_{0}}{\partial t}+\left[f_{0}, \mathcal{K}\right]_{\mathbf{Q}, \mathbf{P}}=0
$$


where the new Hamiltonian $\mathcal{K}$ is a function of the new canonical pair $(\mathbf{Q}, \mathbf{P})$, such that

$$
\mathcal{K}\left(\nabla_{P} S, \mathbf{P} ; t\right)=\frac{\partial S}{\partial t}+H\left(\mathbf{q}, \nabla_{q} S ; t\right),
$$

and the Poisson bracket entering Eq. (23) has the same form as Eq. (3), expressed in the new canonical variables.

\section{PROPAGATION OF AN INTENSE BEAM THROUGH A PERIODIC FOCUSING LATTICE}

As a first application of the Hamiltonian formalism, we consider the propagation of a continuous beam through a periodic focusing lattice in a circular ring with mean radius $R$. Particle motion is accomplished in 2 degrees of freedom in a plane transverse to the design orbit. The model equations consist of the nonlinear Vlasov-Poisson equations [1]

$$
\begin{gathered}
\frac{\partial f}{\partial \theta}+[f, H]_{\mathbf{q}, \mathbf{p}}=0 \\
\nabla_{\mathbf{q}}^{2} \psi=-4 \pi \varrho=-4 \pi \int d^{2} \mathbf{p} f(\mathbf{q}, \mathbf{p} ; \theta),
\end{gathered}
$$

where

$$
\begin{aligned}
H(\mathbf{q}, \mathbf{p} ; \theta)= & \frac{R}{2}\left(p_{x}^{2}+p_{z}^{2}\right)+\frac{1}{2 R}\left(G_{x} x^{2}+G_{z} z^{2}\right) \\
& +\lambda \psi(\mathbf{q} ; \theta)
\end{aligned}
$$

is the normalized Hamiltonian, and $\mathbf{q}=(x, z)$. The transverse canonical momenta $\mathbf{p}=\left(p_{x}, p_{z}\right)$ entering the Hamiltonian (27) are dimensionless variables which represent the actual transverse momenta of the moving particle scaled by the longitudinal momentum of the synchronous particle [12]. The case of a beam propagation in a straight focusing channel is most appropriately described in terms of the path length $s=R \theta$ chosen as an independent variable. Then the Hamiltonian (27) should be divided by the mean radius $R$ and the coefficients $G_{x, z} / R^{2}$ redefined accordingly [12].

In addition, $R$ is the mean radius of the accelerator and $\psi$ is a normalized potential related to the actual electric potential $\varphi$ according to

$$
\psi=\frac{4 \pi \varepsilon_{0}}{N e_{b}} \varphi
$$

where $N$ is the total number of particles in the beam, $e_{b}$ is the particle charge, and $\varepsilon_{0}$ is the electric susceptibility of vacuum. Moreover, the parameter $\lambda$ is defined by

$$
\lambda=\frac{N R r_{b}}{\beta_{s}^{2} \gamma_{s}^{3}},
$$

where $\beta_{s}=v_{s} / c$ is the relative velocity of the synchronous particle, $\gamma_{s}=\left(1-\beta_{s}^{2}\right)^{-1 / 2}$ is the Lorentz factor, and

$$
r_{b}=\frac{e_{b}^{2}}{4 \pi \varepsilon_{0} m_{b} c^{2}}
$$

is the classical radius of a beam particle with charge $e_{b}$ and rest mass $m_{b}$. The coefficients $G_{x, z}(\theta)$ determining the focusing strength in both transverse directions are periodic functions of $\theta$,

$$
G_{x, z}(\theta+\Theta)=G_{x, z}(\theta),
$$

with period $\Theta$.

Following the procedure outlined in the preceding section, we transform Eqs. (25)-(27) according to

$$
\begin{gathered}
{\left[F_{0}, \mathcal{K}\right]_{\mathbf{q}, \mathbf{P}} \equiv 0} \\
\frac{\partial S}{\partial \theta}+\epsilon H\left(\mathbf{q}, \nabla_{\mathbf{q}} S ; \theta\right)=\mathcal{K}(\mathbf{q}, \mathbf{P}), \\
\nabla_{\mathbf{q}}^{2} \psi=-4 \pi \int d^{2} \mathbf{P} F_{0}(\mathbf{q}, \mathbf{P}) \operatorname{det}\left(\nabla_{\mathbf{q}} \nabla_{\mathbf{P}} S\right),
\end{gathered}
$$

where $\epsilon$ is formally a small parameter, which will be set equal to unity at the end of the calculation. Similar to Ref. [8], the small parameter $\epsilon$ is proportional to the applied focusing field. Specifically, the parameter $\epsilon$ scales as $\epsilon \sim \sigma_{v} / 2 \pi<1$, where $\sigma_{v}$ is the vacuum phase advance [8]. Note that all contributions to the original Hamiltonian (27) are allowed to be of the same order of magnitude, where the small parameter $\epsilon$ multiplies $H\left(\mathbf{q}, \nabla_{\mathbf{q}} S ; \theta\right)$ in Eq. (33). Therefore, in this maximal ordering, self-field effects are allowed to be as large as (or weaker than) the applied focusing field. The next step is to expand the quantities $S, \mathcal{K}$, and $\psi$ in a power series in $\epsilon$ according to

$$
\begin{gathered}
S=S_{0}+\epsilon S_{1}+\epsilon^{2} S_{2}+\epsilon^{3} S_{3}+\ldots, \\
\mathcal{K}=\mathcal{K}_{0}+\epsilon \mathcal{K}_{1}+\epsilon^{2} \mathcal{K}_{2}+\epsilon^{3} \mathcal{K}_{3}+\ldots, \\
\psi=\psi_{0}+\epsilon \psi_{1}+\epsilon^{2} \psi_{2}+\epsilon^{3} \psi_{3}+\ldots
\end{gathered}
$$

We now substitute the expansions (35)-(37) into Eqs. (33) and (34) and obtain perturbation equations that can be solved order by order.

The lowest order solution is evident and has the form

$$
\begin{gathered}
S_{0}=\mathbf{q} \cdot \mathbf{P}, \quad \mathcal{K}_{0} \equiv 0, \\
\nabla_{\mathbf{q}}^{2} \psi_{0}=-4 \pi \int d^{2} \mathbf{P} F_{0}(\mathbf{q}, \mathbf{P}) .
\end{gathered}
$$

First-order $O(\epsilon)$. - Taking into account the already obtained lowest-order solutions (38) and (39), the HamiltonJacobi equation (33) to first order in $\epsilon$ can be expressed as

$$
\begin{gathered}
\frac{\partial S_{1}}{\partial \theta}+\frac{R}{2}\left(P_{x}^{2}+P_{z}^{2}\right)+ \\
\frac{1}{2 R}\left(G_{x} x^{2}+G_{z} z^{2}\right)+\lambda \psi_{0}=\mathcal{K}_{1}(\mathbf{q}, \mathbf{P}) .
\end{gathered}
$$


Imposing the condition that the first-order Hamiltonian $\mathcal{K}_{1}$ be equal to

$$
\begin{aligned}
\mathcal{K}_{1}(\mathbf{q}, \mathbf{P})= & \frac{R}{2}\left(P_{x}^{2}+P_{z}^{2}\right)+\frac{1}{2 R}\left(\bar{G}_{x} x^{2}+\bar{G}_{z} z^{2}\right) \\
& +\lambda \psi_{0}(\mathbf{q})
\end{aligned}
$$

we obtain immediately

$$
\begin{gathered}
S_{1}=-\frac{1}{2 R}\left[\tilde{G}_{x}(\theta) x^{2}+\tilde{G}_{z}(\theta) z^{2}\right], \\
\psi_{1} \equiv 0 .
\end{gathered}
$$

Here we introduce the notation

$$
\begin{aligned}
\bar{G}_{x, z} & =\frac{1}{\Theta} \int_{\theta_{0}}^{\theta_{0}+\Theta} d \theta G_{x, z}(\theta) \\
\tilde{G}_{x, z}(\theta) & =\int_{\theta_{0}}^{\theta_{0}+\theta} d \tau\left[G_{x, z}(\tau)-\bar{G}_{x, z}\right] .
\end{aligned}
$$

Note that since the focusing coefficients are periodic functions of $\theta$ they can be expanded in a Fourier series

$$
G_{x, z}(\theta)=\sum_{n=-\infty}^{\infty} G_{x, z}^{(n)} \exp (i n \Omega \theta)
$$

where

$$
G_{x, z}^{(n)}=\frac{1}{\Theta} \int_{0}^{\Theta} d \theta G_{x, z}(\theta) \exp (-i n \Omega \theta),
$$

and $\Omega=2 \pi / \Theta$. Therefore, for the quantities $\bar{G}_{x, z}$ and $\tilde{G}_{x, z}(\theta)$ expressed in terms of the Fourier amplitudes, we obtain

$$
\bar{G}_{x, z}=G_{x, z}^{(0)}, \quad \tilde{G}_{x, z}(\theta)=-\frac{i}{\Omega} \sum_{n \neq 0} \frac{G_{x, z}^{(n)}}{n} \exp (i n \Omega \theta) .
$$

Second-order $O\left(\epsilon^{2}\right)$. - To this order, the HamiltonJacobi equation (33) takes the form

$$
\frac{\partial S_{2}}{\partial \theta}-\left(x P_{x} \tilde{G}_{x}+{ }_{z} P_{z} \tilde{G}_{z}\right)=\mathcal{K}_{2}(\mathbf{q}, \mathbf{P}) .
$$

It is straightforward to solve Eq. (48), yielding the obvious result
$S_{2}=x P_{x} \tilde{\tilde{G}}_{x}(\theta)+z P_{z} \tilde{\tilde{G}}_{z}(\theta), \quad \mathcal{K}_{2}(\mathbf{q}, \mathbf{P}) \equiv 0$.

For the second-order potential $\psi_{2}$ we obtain the equation

$$
\nabla_{\mathbf{q}}^{2} \psi_{2}=-4 \pi\left(\tilde{\tilde{G}}_{x}+\tilde{\tilde{G}}_{z}\right) \int d^{2} \mathbf{P} F_{0}(\mathbf{q}, \mathbf{P})
$$

or, making use of (39),

$$
\psi_{2}(\mathbf{q} ; \theta)=\left[\tilde{\tilde{G}}_{x}(\theta)+\tilde{\tilde{G}}_{z}(\theta)\right] \psi_{0}(\mathbf{q}) .
$$

In Eqs. (49)-(51), $\tilde{\tilde{G}}_{x, z}(\theta)$ denotes application of the integral operation in Eq. (44) to $\tilde{G}_{x, z}(\theta)$, i.e.,

$$
\tilde{\tilde{G}}_{x, z}(\theta)=\int_{\theta_{0}}^{\theta_{0}+\theta} d \tau \tilde{G}_{x, z}(\tau)
$$

because $\overline{\tilde{G}}_{x, z}=0$.

Third-order $O\left(\epsilon^{3}\right)$. - To third order in $\epsilon$, the HamiltonJacobi equation (33) can be written as

$$
\begin{gathered}
\frac{\partial S_{3}}{\partial \theta}+R\left(P_{x}^{2} \tilde{\tilde{G}}_{x}+P_{z}^{2} \tilde{\tilde{G}}_{z}\right)+ \\
\frac{1}{2 R}\left(\tilde{G}_{x}^{2} x^{2}+\tilde{G}_{z}^{2} z^{2}\right)+\lambda\left(\tilde{\tilde{G}}_{x}+\tilde{\tilde{G}}_{z}\right) \psi_{0}=\mathcal{K}_{3}(\mathbf{q}, \mathbf{P}) .
\end{gathered}
$$

The third-order Hamiltonian $\mathcal{K}_{3}$ is given by the expression

$$
\mathcal{K}_{3}(\mathbf{q}, \mathbf{P})=\frac{1}{2 R}\left(\tilde{G}_{x}^{2} x^{2}+\overline{\tilde{G}}_{z}^{2} z^{2}\right)
$$

Equation (53) can be solved easily for the third-order generating function $S_{3}$. The result is

$$
\begin{aligned}
S_{3}= & -R\left(P_{x}^{2} \tilde{\tilde{\tilde{G}}}_{x}+P_{z}^{2} \tilde{\tilde{\tilde{G}}}_{z}\right)-\frac{1}{2 R}\left(\widetilde{\tilde{G}}_{x}^{2} x^{2}+\widetilde{\tilde{G}}_{z}^{2} z^{2}\right) \\
& -\lambda\left(\tilde{\tilde{\tilde{G}}}_{x}+\tilde{\tilde{\tilde{G}}}_{z}\right) \psi_{0} .
\end{aligned}
$$

For the third-order electric potential $\psi_{3}$ we obtain simply

$$
\psi_{3} \equiv 0 \text {. }
$$

Fourth-order $O\left(\epsilon^{4}\right)$. - To the fourth order in the expansion parameter $\epsilon$, the Hamilton-Jacobi equation (33) can be expressed as

$$
\frac{\partial S_{4}}{\partial \theta}-x P_{x}\left(\tilde{\widetilde{G}}_{x}^{2}+\tilde{G}_{x} \tilde{\tilde{G}}_{x}\right)-z P_{z}\left(\tilde{\tilde{G}}_{z}^{2}+\tilde{G}_{z} \tilde{\tilde{G}}_{z}\right)-\lambda R\left(\tilde{\tilde{G}}_{x}+\tilde{\tilde{G}}_{z}\right)\left(P_{x} \frac{\partial \psi_{0}}{\partial x}+P_{z} \frac{\partial \psi_{0}}{\partial z}\right)=\mathcal{K}_{4}(\mathbf{q}, \mathbf{P})
$$

The obvious condition to impose is that the fourth-order Hamiltonian $\mathcal{K}_{4}$ be equal to

$$
\mathcal{K}_{4}(\mathbf{q}, \mathbf{P})=-x P_{x} \overline{\tilde{G}_{x} \tilde{\tilde{G}}_{x}}-{ }_{z} P_{z} \overline{\tilde{G}_{z} \tilde{\tilde{G}}_{z}}
$$

Taking into account Eq. (58), it is straightforward to solve the fourth-order Hamilton-Jacobi equation (57) for $S_{4}$. We obtain

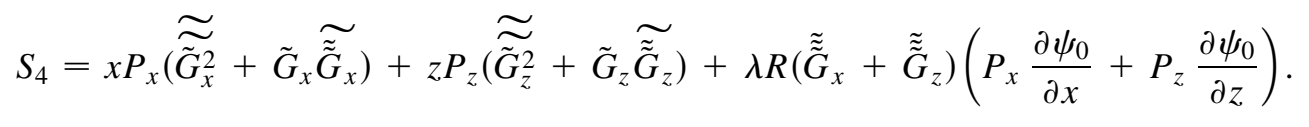

For the fourth-order electric potential $\psi_{4}$, we obtain the Poisson equation

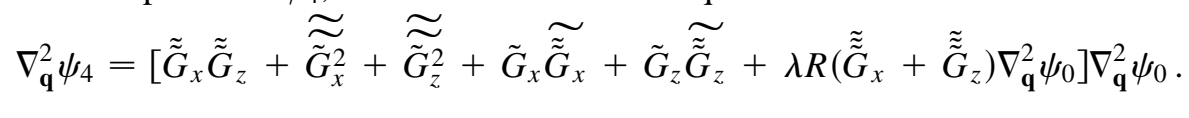


Fifth-order $O\left(\epsilon^{5}\right)$. - In fifth order, we are interested in the Hamiltonian $\mathcal{K}_{5}$. Omitting algebraic details, we find

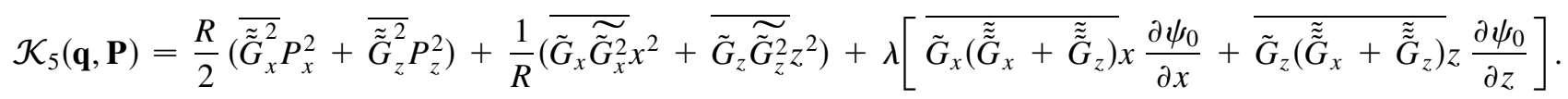

In concluding this section, we collect terms up to fifth order in $\epsilon$ in the new Hamiltonian $\mathcal{K}=\mathcal{K}_{0}+\epsilon \mathcal{K}_{1}+$ $\epsilon^{2} \mathcal{K}_{2}+\ldots$ and set $\epsilon=1$. This gives

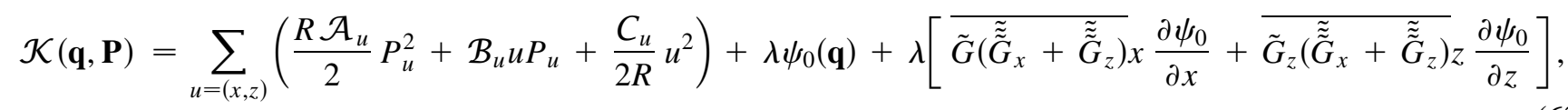

where the coefficients $\mathcal{A}_{u}, \mathcal{B}_{u}$, and $C_{u}$ are defined by the expressions

$$
\begin{aligned}
& \mathcal{A}_{u}=1+\epsilon^{4} \overline{\tilde{\tilde{G}}_{u}^{2}}, \\
& \mathcal{B}_{u}=-\epsilon^{3} \overline{\tilde{G}_{u} \tilde{\tilde{G}}_{u}},
\end{aligned}
$$

and

$$
C_{u}=\bar{G}_{u}+\epsilon^{2} \overline{\tilde{G}}_{u}^{2}+2 \epsilon^{4} \overline{\tilde{G}_{u} \tilde{G}_{u}^{2}} .
$$

The Hamiltonian (62), neglecting the contribution from the self-field $\psi_{0}$, describes the unperturbed betatron oscillations in both the horizontal and the vertical directions.

It is useful to compute the unperturbed betatron tunes $\nu_{x, z}$ in terms of averages over the focusing field strengths.
For a Hamiltonian system governed by a quadratic form in the canonical variables of the type in Eq. (62), it is well known that the characteristic frequencies $\nu_{x, z}$ can be expressed as

$$
\nu_{u}^{2}=\mathcal{A}_{u} C_{u}-\mathcal{B}_{u}^{2} \quad(u=x, z)
$$

Keeping terms up to sixth order in the perturbation parameter $\epsilon$, we obtain

$$
\begin{aligned}
\nu_{u}^{2}= & \bar{G}_{u}+\epsilon^{2} \overline{\tilde{G}}_{u}^{2}+\epsilon^{4}\left(\bar{G}_{u} \overline{\tilde{\tilde{G}}_{u}^{2}}+2 \overline{\tilde{G}_{u} \tilde{\tilde{G}}_{u}^{2}}\right) \\
& +\epsilon^{6}\left[\overline{\tilde{G}_{u}^{2}} \overline{\tilde{\tilde{G}}_{u}^{2}}-\left(\overline{\left.\left.\tilde{G}_{u}^{2} \tilde{\tilde{G}}_{u}\right)^{2}\right] .}\right.\right.
\end{aligned}
$$

In terms of Fourier amplitudes of the focusing coefficients, Eq. (67) can be expressed as

$$
\begin{aligned}
\nu_{u}^{2}= & G_{u}^{(0)}+\frac{2 \epsilon^{2}}{\Omega^{2}} \sum_{n=1}^{\infty} \frac{\left|G_{u}^{(n)}\right|^{2}}{n^{2}} \\
& +\frac{2 \epsilon^{4}}{\Omega^{4}}\left[G_{u}^{(0)} \sum_{n=1}^{\infty} \frac{\left|G_{u}^{(n)}\right|^{2}}{n^{4}}+2 \sum_{\substack{m, n=1 \\
m \neq n}}^{\infty} \frac{\operatorname{Re}\left(G_{u}^{(m) *} G_{u}^{(n)} G_{u}^{(m-n)}\right)}{m n(m-n)^{2}}-2 \sum_{m, n=1}^{\infty} \frac{\operatorname{Re}\left(G_{u}^{(m)} G_{u}^{(n)} G_{u}^{(m+n) *}\right)}{m n(m+n)^{2}}\right] \\
& +\frac{4 \epsilon^{6}}{\Omega^{6}} \sum_{m, n=1}^{\infty} \frac{\left|G_{u}^{(m)}\right|^{2}\left|G_{u}^{(n)}\right|^{2}}{m^{2} n^{4}} .
\end{aligned}
$$

For illustration purposes, we consider a simple FODO lattice with equal focusing and defocusing strengths $+G$ and $-G$ and period $\Theta$. We also assume that the longitudinal dimensions $\theta_{f}$ of the focusing and defocusing lenses are equal; the longitudinal dimensions $\theta_{d}$ of the corresponding drift spaces are assumed to be equal as well. Moreover,

$$
2\left(\theta_{f}+\theta_{d}\right)=\Theta .
$$

For simplicity, we consider the horizontal degree of freedom only (the vertical degree of freedom can be treated in analogous manner). The Fourier amplitudes of the focusing coefficients are

$$
\begin{aligned}
G_{x}^{(2 n+1)} & =\frac{i G}{(2 n+1) \pi}\left\{\exp \left[-i(2 n+1) \Omega \theta_{f}\right]-1\right\}, \\
G_{x}^{(2 n)} & =0,
\end{aligned}
$$

where $n=0,1,2, \ldots$. To second order in $\epsilon$, we obtain for the horizontal betatron tune

$$
\nu_{x}^{2}=\frac{2 \epsilon^{2} \Theta^{2} G^{2}}{\pi^{4}} \sum_{m=1}^{\infty} \frac{1}{(2 m-1)^{4}} \sin ^{2} \frac{(2 m-1) \pi \theta_{f}}{\Theta} .
$$

In the limit of infinitely thin lenses, $\theta_{f} \rightarrow 0$, Eq. (71) reduces to the well-known expression

$$
\nu_{x}^{2}=\frac{\epsilon^{2} \theta_{f}^{2} G^{2}}{4}
$$

where use has been made of the identity

$$
\sum_{m=1}^{\infty} \frac{1}{(2 m-1)^{2}}=\frac{\pi^{2}}{8}
$$

It is evident from Eqs. (68) and (71) that the Hamiltonian averaging technique developed here represents a powerful formalism for evaluating the betatron tunes in terms of averages over the focusing field strength. The analysis in this section has been carried out to sixth order to demonstrate the ease and flexibility of the mixed-variable 
approach. In some practical applications, a description to lower order (e.g. fourth order) may be adequate.

\section{COHERENT BEAM-BEAM INTERACTION}

As a second application of the Hamiltonian formalism developed in Sec. II, we study here the evolution of two counterpropagating beams, nonlinearly coupled by the electromagnetic interaction between the beams at collision. For simplicity, we consider one-dimensional motion in the vertical $(q)$ direction, described by the nonlinear VlasovPoisson equations

$$
\begin{gathered}
\frac{\partial f_{k}}{\partial \theta}+\left[f_{k}, H_{k}\right]=0 \\
\frac{\partial^{2} V_{k}}{\partial q^{2}}=4 \pi \int d p f_{3-k}(q, p ; \theta)
\end{gathered}
$$

where

$$
H_{k}=\frac{\nu_{k}}{2}\left(p^{2}+q^{2}\right)+\lambda_{k} \delta_{p}(\theta) V_{k}(q ; \theta)
$$

is the Hamiltonian. Here $\lambda_{k}$ is the beam-beam coupling parameter, defined according to [13]

$$
\lambda_{k}=\frac{R r_{e} N_{3-k} \beta_{k q}^{*}}{\gamma_{k 0} L_{(3-k) x}} \frac{1+\beta_{k 0} \beta_{(3-k) 0}}{\beta_{k 0}^{2}} \approx \frac{2 R r_{e} N_{3-k} \beta_{k q}^{*}}{\gamma_{k 0} L_{(3-k) x}} .
$$

Moreover, $(k=1,2)$ labels the beam, $f_{k}(q, p ; \theta)$ is the distribution function, $\theta$ is the azimuthal angle, and $\nu_{k}$ is the betatron frequency in the vertical direction. In addition, $R$ is the mean machine radius, $r_{e}$ is the classical electron radius, $N_{1,2}$ is the total number of particles in either beam, $V_{k}(q ; \theta)$ is the normalized beam-beam potential, $\beta_{k q}^{*}$ is the vertical beta function at the interaction point, and $L_{k x}$ is the horizontal dimension of the beam ribbon [14].

Our goal is to determine a canonical transformation such that the new Hamiltonian is time independent. As a consequence, the stationary solution of the Vlasov equation (21) is expressed as a function of the new Hamiltonian. Following the procedure outlined in the Sec. II we transform Eqs. (74)-(76) according to

$$
\begin{gathered}
{\left[F_{0}^{(k)}, \mathcal{K}_{k}\right] \equiv 0} \\
\frac{\partial S_{k}}{\partial \theta}+\epsilon H_{k}\left(q, \frac{\partial S_{k}}{\partial q} ; \theta\right)=\mathcal{K}_{k}(q, P), \\
\frac{\partial^{2} V_{k}}{\partial q^{2}}=4 \pi \int d P \frac{\partial^{2} S_{k}}{\partial q \partial P} F_{0}^{(3-k)}(q, P),
\end{gathered}
$$

where $\epsilon$ is again a formal small parameter, which will be set equal to unity at the end of the calculation. In this case, the small parameter $\epsilon$ scales as the beam-beam parameter [13] $\tilde{\lambda}_{k}$ with $\epsilon \sim \tilde{\lambda}_{k}=(2 / \pi)^{1 / 2} \lambda_{k} \sigma_{k} /\left[\sigma_{3-k}\left(\sigma_{k}^{2}+\right.\right.$ $\left.\left.\sigma_{3-k}^{2}\right)^{1 / 2}\right]$.
The next step is to expand the quantities $S_{k}, \mathcal{K}_{k}$, and $V_{k}$ in a power series in $\epsilon$, analogous to Eqs. (35)-(37), according to

$$
\begin{gathered}
S_{k}=q P+\epsilon G_{k}^{(1)}+\epsilon^{2} G_{k}^{(2)}+\epsilon^{3} G_{k}^{(3)}+\ldots, \\
\mathcal{K}_{k}=\epsilon \mathcal{K}_{k}^{(1)}+\epsilon^{2} \mathcal{K}_{k}^{(2)}+\epsilon^{3} \mathcal{K}_{k}^{(3)}+\ldots, \\
V_{k}=\tilde{V}_{k}+\epsilon V_{k}^{(1)}+\epsilon^{2} V_{k}^{(2)}+\epsilon^{3} V_{k}^{(3)}+\ldots
\end{gathered}
$$

where

$$
\frac{\partial^{2} \tilde{V}_{k}}{\partial q^{2}}=4 \pi \int d P F_{0}^{(3-k)}(q, P)
$$

Substitution of the above expansions (81)-(83) into Eqs. (79) and (80) yields perturbation equations that can be solved successively order by order. The results to third order in $\epsilon$ are briefly summarized below.

First-order $O(\epsilon)$ :

$$
\begin{gathered}
\mathcal{K}_{k}^{(1)}(q, P)=\frac{\nu_{k}}{2}\left(P^{2}+q^{2}\right)+\frac{\lambda_{k}}{2 \pi} \tilde{V}_{k}(q), \\
G_{k}^{(1)}(q, P ; \theta)=\frac{i \lambda_{k}}{2 \pi} \tilde{V}_{k}(q) \sum_{n \neq 0} \frac{1}{n} \exp (i n \theta), \\
V_{k}^{(1)}(q ; \theta) \equiv 0 .
\end{gathered}
$$

Second-order $O\left(\epsilon^{2}\right)$ :

$$
\begin{gathered}
\mathcal{K}_{k}^{(2)}(q, P) \equiv 0 \\
G_{k}^{(2)}(q, P ; \theta)=-\frac{\lambda_{k} \nu_{k}}{2 \pi} P \tilde{V}_{k}^{\prime}(q) \sum_{n \neq 0} \frac{1}{n^{2}} \exp (i n \theta), \\
V_{k}^{(2)}(q ; \theta)=-\frac{\lambda_{k} \nu_{k}}{2 \pi} \tilde{V}_{k}^{(2)}(q) \sum_{n \neq 0} \frac{1}{n^{2}} \exp (i n \theta)
\end{gathered}
$$

where

$$
\frac{\partial^{2} \tilde{V}_{k}^{(2)}}{\partial q^{2}}=4 \pi \tilde{V}_{k}^{\prime \prime}(q) \int d P F_{0}^{(3-k)}(q, P) .
$$

Third-order $O\left(\epsilon^{3}\right)$. - In third order we are interested in the new Hamiltonian, which is of the form

$$
\mathcal{K}_{k}^{(3)}(q, P)=\frac{\lambda_{k}^{2} \nu_{k}}{4 \pi^{2}} \zeta(2)\left[\tilde{V}_{k}^{\prime 2}(q)-2 \tilde{V}_{k}^{(2)}(q)\right],
$$

where $\zeta(z)$ is Riemann's zeta function [15]

$$
\zeta(z)=\sum_{n=1}^{\infty} \frac{1}{n^{z}} .
$$

The effectiveness of the Hamiltonian formalism developed in this paper has been demonstrated in two particular examples. In the first example, discussed in the previous 
section, the short-scale dynamics is contained in the external focusing force acting on the beam, while an essential feature of the coherent beam-beam interaction treated in this section is the relatively fast variation of the collective interaction between the two colliding beams. The simplicity in applying the Hamiltonian averaging technique is embedded in the use of mixed canonical variables. Instead of expanding the generating function and the new Hamiltonian in terms of the new canonical coordinates and momenta $[7,8]$, one has to simply solve the Hamilton-Jacobi equations order by order. It should be pointed out that the mixed variable Hamiltonian formalism can be used to derive amplitude equations, describing processes of formation of patterns and coherent structures in a number of plasma and beam systems in which collective processes are important.

\section{THE EQUILIBRIUM DISTRIBUTION FUNCTION}

Since the new Hamiltonian $\mathcal{K}_{k}$ is time independent (by construction), the equilibrium distribution function $F_{0}^{(k)}$ [see Eq. (78)] is a function of the new Hamiltonian

$$
F_{0}^{(k)}(q, P)=G_{k}\left(\mathcal{K}_{k}\right),
$$

where

$$
\begin{aligned}
\mathcal{K}_{k}(q, P)= & \frac{\nu_{k}}{2}\left(P^{2}+q^{2}\right)+\frac{\lambda_{k}}{2 \pi} \tilde{V}_{k}(q) \\
& +\frac{\lambda_{k}^{2} \nu_{k}}{4 \pi^{2}} \zeta(2)\left[\tilde{V}_{2}^{\prime 2}(q)-2 \tilde{V}_{k}^{(2)}(q)\right] .
\end{aligned}
$$

Integrating Eq. (94) over $P$ we obtain a nonlinear integral equation of the Haissinski type [16] for the equilibrium beam density profile $\varrho_{0}^{(k)}$

$$
\varrho_{0}^{(k)}(q)=\int d P G_{k}\left(\mathcal{K}_{k}\right)
$$

where

$$
\begin{aligned}
\mathcal{K}_{k}(q, P)= & \frac{\nu_{k}}{2}\left(P^{2}+q^{2}\right) \\
& +\lambda_{k} \int d q^{\prime}\left|q-q^{\prime}\right| \varrho_{0}^{(3-k)}\left(q^{\prime}\right) \\
& +2 \lambda_{k}^{2} \nu_{k} \zeta(2) \mathcal{F}_{k}(q), \\
\mathcal{F}_{k}(q)= & \int d q^{\prime} d q^{\prime \prime} Z\left(q-q^{\prime}, q^{\prime}-q^{\prime \prime}\right) \\
& \times \varrho_{0}^{(3-k)}\left(q^{\prime}\right) \varrho_{0}^{(3-k)}\left(q^{\prime \prime}\right), \\
\mathcal{Z}(u, v)= & \operatorname{sgn}(u) \operatorname{sgn}(v)-2|u| \delta(v) .
\end{aligned}
$$

Here, $\operatorname{sgn}(z)$ is the well-known signum function.

Let us further specify the function $G_{k}\left(\mathcal{K}_{k}\right)$ and assume that it is given by the thermal equilibrium distribution $[1,12,17]$

$$
G_{k}\left(\mathcal{K}_{k}\right)=\mathcal{N}_{k} \exp \left(-\frac{\mathcal{K}_{k}}{\varepsilon_{k}}\right)
$$

where $\mathcal{N}_{k}$ is a normalization constant, defined according to

$$
\frac{1}{\mathcal{N}_{k}}=\int d q d P \exp \left[-\frac{\mathcal{K}_{k}(q, P)}{\varepsilon_{k}}\right]
$$

and $\varepsilon_{k}$ is the unnormalized beam emittance. The second term in the Hamiltonian (97) can be transformed according to

$$
\begin{aligned}
\int_{-\infty}^{\infty} d q^{\prime}\left|q-q^{\prime}\right| \varrho_{0}^{(3-k)}\left(q^{\prime}\right) & =q-\left\langle q_{3-k}\right\rangle+2 \int_{q}^{\infty} d q^{\prime}\left(q^{\prime}-q\right) \varrho_{0}^{(3-k)}\left(q^{\prime}\right) \\
& =q-\left\langle q_{3-k}\right\rangle+2 \int_{0}^{\infty} d q_{1} q_{1} \varrho_{0}^{(3-k)}\left(q_{1}+q\right)
\end{aligned}
$$

where

$$
\left\langle q_{k}^{n}\right\rangle=\int_{-\infty}^{\infty} d q q^{n} \varrho_{0}^{(k)}(q) .
$$

Expanding the beam density $\varrho_{0}^{(3-k)}\left(q_{1}+q\right)$ occurring in the integral in Eq. (102) in a Taylor series and integrating by parts, we obtain

$$
\int_{-\infty}^{\infty} d q^{\prime}\left|q-q^{\prime}\right| \varrho_{0}^{(3-k)}\left(q^{\prime}\right)=\left\langle q_{3-k}^{(+)}\right\rangle-\left\langle q_{3-k}^{(-)}\right\rangle+\left(1-2 \mathcal{A}_{3-k}\right) q+2 \sum_{n=2}^{\infty} \frac{C_{3-k}^{(n)}}{n !} q^{n},
$$

where

$$
\begin{aligned}
& \left\langle q_{k}^{(+)}\right\rangle=\int_{0}^{\infty} d q q \varrho_{0}^{(k)}(q), \\
& \left\langle q_{k}^{(-)}\right\rangle=\int_{-\infty}^{0} d q q \varrho_{0}^{(k)}(q),
\end{aligned}
$$

$$
\begin{aligned}
\mathcal{A}_{k} & =\int_{0}^{\infty} d q \varrho_{0}^{(k)}(q), \\
C_{k}^{(n)} & =\left.\frac{\partial^{n-2} \varrho_{0}^{(k)}(q)}{\partial q^{n-2}}\right|_{q=0} .
\end{aligned}
$$

Substituting Eqs. (100) and (104) into Eq. (96), we obtain 


$$
\varrho_{0}^{(k)}(q)=\mathcal{N}_{k} \sqrt{\frac{2 \pi \varepsilon_{k}}{\nu_{k}}} \exp \left[-\frac{\nu_{k} q^{2}}{2 \varepsilon_{k}}-\frac{\lambda_{k}}{\varepsilon_{k}}\left(1-2 \mathcal{A}_{3-k}\right) q-\frac{2 \lambda_{k}}{\varepsilon_{k}} \sum_{n=2}^{\infty} \frac{C_{3-k}^{(n)}}{n !} q^{n}\right]
$$

Taking into account that

$$
\begin{aligned}
\mathcal{A}_{k}= & \frac{\pi \varepsilon_{k}}{\nu_{k}} \mathcal{N}_{k}+O\left(\lambda_{k}\right), \\
C_{k}^{(n)}= & \mathcal{N}_{k} \sqrt{\frac{2 \pi \varepsilon_{k}}{\nu_{k}}}(-1)^{n-2}\left(\frac{\nu_{k}}{2 \varepsilon_{k}}\right)^{(n-2) / 2} \\
& \times H_{n-2}(0)+O\left(\lambda_{k}\right),
\end{aligned}
$$

where $H_{n}(z)$ is the Hermite polynomial [15] of order $n$, we obtain

$$
\varrho_{0}^{(k)}(q)=\mathcal{N}_{k} \sqrt{\frac{2 \pi \varepsilon_{k}}{\nu_{k}}} \exp \left[h_{k}(q)\right]
$$

where

$$
\begin{aligned}
h_{k}(q)= & -\frac{\nu_{k} q^{2}}{2 \varepsilon_{k}}-\frac{\lambda_{k} \mathcal{B}_{3-k} q}{\varepsilon_{k}} \\
& -\frac{2 \pi \lambda_{k} \varepsilon_{3-k} \mathcal{N}_{3-k}}{\varepsilon_{k} \nu_{3-k}}\left[q \Phi\left(a_{3-k} q\right)+\frac{e^{-a_{3-k}^{2} q^{2}}}{a_{3-k} \sqrt{\pi}}\right],
\end{aligned}
$$

and

$$
\mathcal{B}_{k}=1-\frac{2 \pi \varepsilon_{k} \mathcal{N}_{k}}{\nu_{k}}, \quad a_{k}^{2}=\frac{\nu_{k}}{2 \varepsilon_{k}} .
$$

Here, $\Phi(z)$ is the error function [15].

In order to determine the normalization constant(s), $\mathcal{N}_{k}$, we utilize the method of Laplace to take the integral of the beam density $\varrho_{0}^{(k)}(q)$ over $q$. The first step consists of finding the extremum value(s) $q_{k}^{(e)}$ of the function(s) $h_{k}(q)$. These values satisfy the (two) equation(s)

$$
\begin{gathered}
\frac{\nu_{k} q_{k}^{(e)}}{\varepsilon_{k}}+\frac{\lambda_{k} \mathcal{B}_{3-k}}{\varepsilon_{k}}+ \\
\frac{2 \pi \lambda_{k} \varepsilon_{3-k} \mathcal{N}_{3-k}}{\varepsilon_{k} \nu_{3-k}} \Phi\left(a_{3-k} q_{k}^{(e)}\right)=0 .
\end{gathered}
$$

These are evidently maxima since

$$
\begin{aligned}
h_{k}^{\prime \prime}\left(q_{k}^{(e)}\right) & =-\frac{\nu_{k}}{\varepsilon_{k}}-\frac{4 \pi \lambda_{k} \varepsilon_{3-k} \mathcal{N}_{3-k}}{\varepsilon_{k} \nu_{3-k}} \frac{a_{3-k}}{\sqrt{\pi}} e^{-a_{3-k}^{2} q_{k}^{(e) 2}} \\
& <0 .
\end{aligned}
$$

Integrating the beam density (109) over $q$, we obtain [18]

$$
1=2 \pi \mathcal{N}_{k} \sqrt{\frac{\varepsilon_{k}}{\nu_{k}\left|h_{k}^{\prime \prime}\left(q_{k}^{(e)}\right)\right|}} \exp \left[h_{k}\left(q_{k}^{(e)}\right)\right] .
$$

Equation (114) represents two transcendental equations for determining the normalization constants $\mathcal{N}_{k}$. For the beam centroid and the beam size, i.e., the first and second moments of the beam density (109), we obtain

$$
\left\langle q_{k}\right\rangle=q_{k}^{(e)}+\frac{2 \mathcal{N}_{k}}{\left|h_{k}^{\prime \prime}\left(q_{k}^{(e)}\right)\right|} \sqrt{\frac{2 \pi \varepsilon_{k}}{\nu_{k}}} \exp \left[h_{k}\left(q_{k}^{(e)}\right)\right]
$$

$$
\begin{aligned}
\left\langle q_{k}^{2}\right\rangle= & q_{k}^{(e) 2}+\frac{4 \mathcal{N}_{k} q_{k}^{(e)}}{\left|h_{k}^{\prime \prime}\left(q_{k}^{(e)}\right)\right|} \sqrt{\frac{2 \pi \varepsilon_{k}}{\nu_{k}}} \exp \left[h_{k}\left(q_{k}^{(e)}\right)\right] \\
& +2 \pi \mathcal{N}_{k} \sqrt{\frac{\varepsilon_{k}}{\nu_{k}}}\left[\frac{1}{\left|h_{k}^{\prime \prime}\left(q_{k}^{(e)}\right)\right|}\right]^{3 / 2} \exp \left[h_{k}\left(q_{k}^{(e)}\right)\right] .
\end{aligned}
$$

In order to proceed further, we assume that the beambeam coupling parameter $\lambda_{k}$ is small, and expand the equilibrium beam density $\varrho_{0}^{(k)}(q)$ in a perturbation series in $\lambda_{k}$ according to

$$
\varrho_{0}^{(k)}(q)=\varrho_{00}^{(k)}(q)+\lambda_{k} \varrho_{01}^{(k)}(q)+\ldots,
$$

where

$$
\varrho_{00}^{(k)}(q)=\frac{\mathcal{N}_{k} \sqrt{\pi}}{a_{k}} \exp \left(-a_{k}^{2} q^{2}\right)
$$

and

$$
\begin{aligned}
\varrho_{01}^{(k)}(q)=-\frac{1}{\varepsilon_{k}}\{ & \mathcal{B}_{3-k} q+\frac{\pi \mathcal{N}_{3-k}}{a_{3-k}^{2}} \\
& \left.\times\left[q \Phi\left(a_{3-k} q\right)+\frac{e^{-a_{3-k}^{2} q^{2}}}{a_{3-k} \sqrt{\pi}}\right]\right\} \varrho_{00}^{(k)}(q) .
\end{aligned}
$$

The main goal in what follows is to determine the normalization constant(s) $\mathcal{N}_{k 0}$. To do so we integrate Eq. (117) over $q$. As a result of simple algebraic manipulations, we obtain

$\frac{\pi \mathcal{N}_{k}}{a_{k}^{2}}-\frac{\pi \sqrt{\pi} \lambda_{k}}{\varepsilon_{k} a_{k}^{3} a_{3-k}^{3}} \sqrt{a_{k}^{2}+a_{3-k}^{2}} \mathcal{N}_{k} \mathcal{N}_{3-k}=1$.

Introducing the new unknowns

$$
\mathcal{M}_{k}=\frac{\pi \mathcal{N}_{k}}{a_{k}^{2}},
$$

we can write the two equations for determining $\mathcal{M}_{1,2}$ as

$$
1=\mathcal{M}_{1}-b_{1} \mathcal{M}_{1} \mathcal{M}_{2}, \quad 1=\mathcal{M}_{2}-b_{2} \mathcal{M}_{1} \mathcal{M}_{2},
$$

where

$$
b_{1}=\frac{\lambda_{1}}{\varepsilon_{1} \sqrt{\pi}} \frac{\sqrt{a_{1}^{2}+a_{2}^{2}}}{a_{1} a_{2}}, \quad b_{2}=\frac{\lambda_{2}}{\varepsilon_{2} \sqrt{\pi}} \frac{\sqrt{a_{1}^{2}+a_{2}^{2}}}{a_{1} a_{2}} .
$$

From Eq. (122), as a result of simple algebraic manipulations we obtain the quadratic equation

$$
b_{2} \mathcal{M}_{1}^{2}-\left(b_{2}-b_{1}+1\right) \mathcal{M}_{1}+1=0
$$

for $\mathcal{M}_{1}$, and the equation

$$
b_{1} \mathcal{M}_{2}=b_{2} \mathcal{M}_{1}+b_{1}-b_{2}
$$


for determining $\mathcal{M}_{2}$ once $\mathcal{M}_{1}$ is known. Equation (124) has one real double root if and only if the discriminant

$$
\mathcal{D}=\left(b_{2}-b_{1}+1\right)^{2}-4 b_{2}
$$

is equal to zero. This gives

$$
b_{2}=\left(\sqrt{b_{1}} \pm 1\right)^{2} .
$$

Since the scaled normalization constants $\mathcal{M}_{1,2}$ should be positive, we choose

$$
b_{2}=\left(\sqrt{b_{1}}-1\right)^{2} .
$$

Thus we obtain

$$
\mathcal{M}_{1}=\frac{1}{\sqrt{b_{2}}}=\frac{1}{\left|\sqrt{b_{1}}-1\right|}, \quad \mathcal{M}_{2}=\frac{1}{\sqrt{b_{1}}} .
$$

To conclude this section we note that in the case of $\mathcal{D} \neq 0$ we have two solutions for either $\mathcal{M}$, i.e.,

$$
\begin{aligned}
& \mathcal{M}_{1}^{(1,2)}=\frac{b_{2}-b_{1}+1 \pm \sqrt{\mathcal{D}}}{2 b_{2}}, \\
& \mathcal{M}_{2}^{(1,2)}=\frac{b_{1}-b_{2}+1 \pm \sqrt{\mathcal{D}}}{2 b_{1}} .
\end{aligned}
$$

Note also that the discriminant $\mathcal{D}$ is invariant (does not change) under permutation of $b_{1}$ and $b_{2}$. In other words, four different physically realizable situations are possible for a wide range of parameters

$$
0<b_{2}<1+b_{1}
$$

The inequality in Eq. (131) was obtained under the condition that both solutions in Eq. (130) are positive. This case corresponds to the so-called "flip-flop" state [19] of the two colliding beams, which is a bifurcated state that is better to be avoided.

\section{CONCLUSIONS}

We developed a systematic canonical perturbation approach that removes rapidly oscillating terms in Hamiltonians of quite general form. The essential feature of this approach is the use of mixed canonical variables. For this purpose the Vlasov-Poisson equations are transformed to mixed canonical variables, and an appropriate perturbation scheme is chosen to obtain the equilibrium phase space density. It is worthwhile to note that the perturbation expansion outlined in the preceding section can be carried out to arbitrary order, although higher-order calculations become very tedious.

In conclusion, it is evident from the present analysis that the Hamiltonian averaging technique developed here represents a powerful formalism with applications ranging from beam propagation through a periodic focusing lattice (Sec. III) to coherent beam-beam interaction (Secs. IV and V). For example, in the application to the coherent beam-beam interaction, the rapidly oscillating terms due to the periodic beam-beam kicks have been averaged away, leading to a new time-independent Hamiltonian (Sec. IV). Furthermore, the equilibrium distribution functions have been obtained as a general function of the new Hamiltonian, and a coupled set of integral equations for the beam densities has been obtained (Sec. V). An intriguing feature of the analysis in Sec. V is the derivation of a condition for the existence of the so-called flip-flop state [19] of the two colliding beams, which is a bifurcated state that is better to be avoided in experimental applications.

We reiterate that the formalism developed here is, strictly speaking, noncanonical and, in general, does not provide complete elimination of fast oscillating terms in the transformed Vlasov equation in the mixed variables. Nevertheless, such an elimination can be performed in the new Hamiltonian in the mixed variables. Moreover, if the distribution function is assumed to be an arbitrary function of the new time-independent Hamiltonian, it is a stationary solution of the nonlinear Vlasov equation in the mixed variables.

Finally, we reiterate that the mixed variable Hamiltonian formalism developed in the present analysis can be used to derive amplitude equations, describing processes of formation of patterns and coherent structures in a number of plasma and beam systems in which collective processes are important.

\section{ACKNOWLEDGMENTS}

We are indebted to S. A. Heifets for many fruitful discussions concerning the subject of this paper. It is a pleasure to thank H. Qin for illuminating discussions and comments. This research was supported by the U.S. Department of Energy.

[1] R. C. Davidson and H. Qin, Physics of Intense Charged Particle Beams in High Energy Accelerators (World Scientific, Singapore, 2001), and references therein.

[2] A. W. Chao, Physics of Collective Beam Instabilities in High Energy Accelerators (Wiley, New York, 1993).

[3] S. I. Tzenov, Fermilab Report No. FERMILAB-Pub98/287, 1998.

[4] Y. Cai, A. W. Chao, S. I. Tzenov, and T. Tajima, Phys. Rev. ST Accel. Beams 4, 011001 (2001).

[5] A. W. Chao, P. Bambade, and W. T. Weng, in Proceedings of the US-CERN School on Particle Accelerators, Santa Margherita di Pula, Sardinia, 1985, edited by J. M. Jowett, M. Month, and S. Turner, Lecture Notes in Physics Vol. 247 (Springer-Verlag, Berlin, 1986), p. 77.

[6] J. T. Seeman, in Proceedings of the US-CERN School on Particle Accelerators, Santa Margherita di Pula, Sardinia, 1985 (Ref. [5]), p. 121.

[7] P. J. Channell, Phys. Plasmas 6, 982 (1999).

[8] R. C. Davidson, H. Qin, and P. J. Channell, Phys. Rev. ST Accel. Beams 2, 074401 (1999); 3, 029901 (2000). 
[9] A. J. Lichtenberg and M. A. Lieberman, Regular and Stochastic Motion (Springer-Verlag, Berlin, 1983).

[10] S.I. Tzenov and R.C. Davidson, in Proceedings of the 2001 Particle Accelerator Conference, Chicago (to be published).

[11] J. A. Ellison and R. L. Warnock, SLAC Report No. SLACPUB-8778, 2000.

[12] S. I. Tzenov, Contemporary Accelerator Physics (to be published).

[13] S. I. Tzenov and R. C. Davidson, in Proceedings of the 2001 Particle Accelerator Conference, Chicago (Ref. [10]).

[14] A. W. Chao and R. D. Ruth, Part. Accel. 16, 201 (1985).
[15] M. Abramowitz and I. A. Stegun, Handbook of Mathematical Functions with Formulas Graphs and Mathematical Tables (Wiley, New York, 1984).

[16] J. Haissinski, Nuovo Cimento Soc. Ital. Fis. 18B, 72 (1973).

[17] Yu. L. Klimontovich, Statistical Physics (Harwood Academic, Chur, Switzerland, 1986).

[18] A.H. Nayfeh, Introduction to Perturbation Techniques (Wiley, New York, 1981).

[19] A. V. Otboyev and E. A. Perevedentsev, Phys. Rev. ST Accel. Beams 2, 104401 (1999). 